\title{
A novel adaptor system enables endovascular access through extracorporeal life support circuits
}

Chetan Pasrija, MD, ${ }^{\text {a }}$ Gregory J. Bittle, MD, ${ }^{\mathrm{a}}$ Jiafeng Zhang, MS, ${ }^{\mathrm{a}}$ David Morales, MD, ${ }^{\mathrm{a}}$ Douglas Tran, BS, ${ }^{\mathrm{a}}$ Kristopher B. Deatrick, MD, ${ }^{a}$ James S. Gammie, MD, ${ }^{a}$ Zhongjun Wu, PhD, ${ }^{a}$ Bartley P. Griffith, MD, ${ }^{a}$ Zachary N. Kon, MD, ${ }^{b}$ and David J. Kaczorowski, MD $^{\mathrm{a}}$

\section{ABSTRACT}

Objective: Extracorporeal life support has traditionally been used as a supportive platform for patients with cardiopulmonary failure. Many of these patients require endovascular access for the performance of diagnostic or therapeutic procedures, and obtaining vascular access in these patients can be problematic. We sought to develop a novel system that allows the extracorporeal life support circuit to serve as an access point to the cardiovascular system.

Methods: By using computer-aided design, modeling, and 3-dimensional printing, a novel adaptor that can be easily inserted and removed from an extracorporeal life support circuit was developed. A mock loop was used to measure flow and pressure at various pump speeds with insertion of guidewires and catheters through the adaptor. The ability of the system to enable performance of endovascular procedures in vivo was then tested in a porcine extracorporeal life support model.

Results: By using a small arterial cannula (15F) at 3500 RPM and 3.2 LPM, 15\% and $24 \%$ decrements in circuit flow were observed when a 0.035 " guidewire and $5 \mathrm{~F}$ angiography catheter, respectively, were passed through the adaptor $(P<.001)$. However, when using a larger arterial cannula $(23 \mathrm{~F})$ at 3500 RPM and 4.7 LPM, only $3 \%$ and $5 \%$ decrements in flow were observed $(P<.001)$, respectively, with intermediate changes when using $17 \mathrm{~F}$ to $21 \mathrm{~F}$ cannulas. In vivo testing confirmed that this system enables the performance of a variety of endovascular procedures, including left ventriculography, aortic root and coronary angiography, and descending aortography.

Conclusions: This novel system successfully enables endovascular access through an extracorporeal life support circuit. This technology may transform extracorporeal life support from a purely supportive strategy to a platform for endovascular intervention. (J Thorac Cardiovasc Surg 2019;158:1359-66)

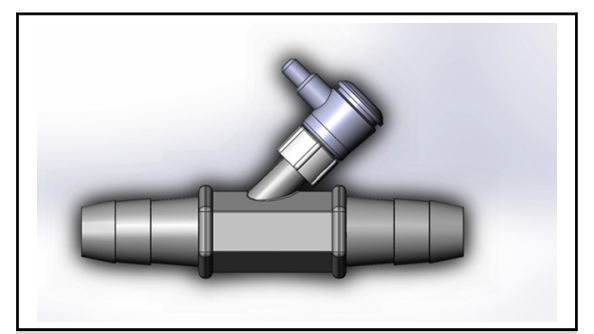

A novel adaptor system that can be easily inserted and removed from an ECLS circuit.

Central Message

A novel adaptor system that can be easily inserted and removed from a circuit can successfully enable endovascular access through an ECLS circuit.

\section{Perspective}

A novel adaptor system enables endovascular access through an ECLS circuit without a clinically significant decrement in flow. This may transform ECLS from a purely supportive strategy to a platform for endovascular intervention.

See Commentaries on pages 1367 and 1368.
Since its first clinical application in 1972, extracorporeal membrane oxygenation (ECMO) and other forms of extracorporeal life support (ECLS) have been used to successfully support patients with life-threatening cardiac or

\footnotetext{
From the a Division of Cardiac Surgery, University of Maryland School of Medicine, Baltimore, Md; and ${ }^{\mathrm{b}}$ New York University Langone Health, New York, NY.

Funding was provided by the Department of Surgery at the University of Maryland School of Medicine.

Received for publication Oct 9, 2018; revisions received Jan 25, 2019; accepted for publication Feb 9, 2019; available ahead of print March 20, 2019.

Address for reprints: David J. Kaczorowski, MD, Division of Cardiac Surgery, University of Maryland, 110 S. Paca St, 7th Floor, Baltimore, MD 21201 (E-mail: Dkaczorowski@som.umaryland.edu).

$0022-5223 / \$ 36.00$

Copyright (c) 2019 by The American Association for Thoracic Surgery https://doi.org/10.1016/j.jtcvs.2019.02.041
}

pulmonary failure. ${ }^{1}$ A number of technologic advances in all components of the ECLS circuit have occurred since the time of its introduction. For example, improved cannula design has allowed more facile insertion with less trauma to blood vessels. Advances in pump and oxygenator design have allowed for greater efficiency and less trauma to blood elements. ${ }^{2}$ These improvements, along with advances in the clinical management of patients requiring ECLS and the

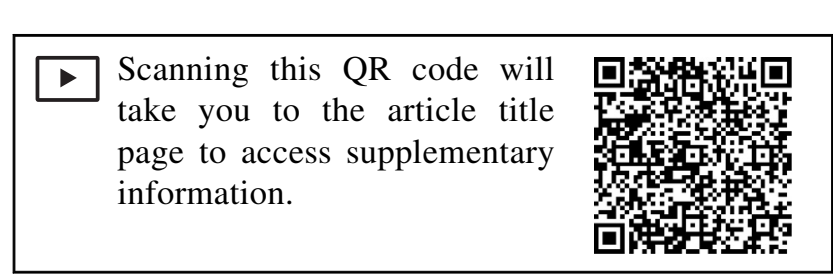




\section{Abbreviations and Acronyms \\ $\mathrm{CFD}=$ computational fluid dynamics \\ ECLS = extracorporeal life support \\ $\mathrm{ECMO}=$ extracorporeal membrane oxygenation \\ PCI = percutaneous coronary intervention}

observation that ECMO could be lifesaving in patients with ARDS due to H1N1 influenza during the 2009 pandemic, ${ }^{3}$ have ignited a rapid increase in the use of ECLS in recent years. $^{4-6}$

To date, ECMO and other forms of ECLS have generally been used as solely supportive technologies, with the intent of providing hemodynamic stability or gas exchange until recovery occurs. However, many patients who require ECLS also require procedures for diagnosis and potential intervention. Many of these procedures, such as left and right heart catheterization, percutaneous coronary intervention (PCI), or insertion of catheters for instillation of thrombolytics, require access to the cardiovascular system. However, vascular access in patients on ECLS can be problematic. Landmarks may be obscured, rendering access more difficult. Blood vessels may be vasoconstricted because of high doses of vasopressors or as a consequence of the shock state preceding cannulation and institution of ECLS. Anticoagulation used with ECLS or coagulopathy associated with the underlying disease state may result in bleeding complications upon insertion or removal of vascular access catheters. Furthermore, vascular access may be limited by the presence of mechanical support devices and monitoring lines. For example, a patient may have 1 femoral artery occupied by an arterial cannula and the other occupied by a balloon pump or temporary left ventricular assist device. On the basis of these observations, we sought to develop a novel system that would safely allow the ECLS system itself to serve as an access point to the cardiovascular system.

\section{MATERIALS AND METHODS \\ Prototype Development}

We sought to develop a system to allow endovascular access through an ECLS circuit with the following characteristics: (1) facile insertion into and removal from an ECLS circuit; (2) the ability to allow hemostatic, reversible passage of wires and catheters into an ECLS circuit that is directed toward the patient by the system; (3) the ability to de-air the system; (4) a mechanism to prevent stagnant flow of blood when the system is in place during prolonged support; and (5) absence of any permanent valves, irregular surfaces, or obstruction to flow that might result in thrombus formation during prolonged support. After prototypes were designed, 3-dimensional printing (stereolithography, Form 2 Printer, Grey Pro Photopolymer Resin, Formlabs, Inc, Somerville, Mass) was used to create the system for use in testing (PRG Prototyping, Pittsburg, Kan). The system consists of a cylindrical tube with tapered ends that may be reversibly inserted into 3/8-inch ECLS tubing. An angled side port interfaces with a hemostatic cap that allows passage of wires and catheters, as well as de-airing (Figure 1, $A$ and $B$,

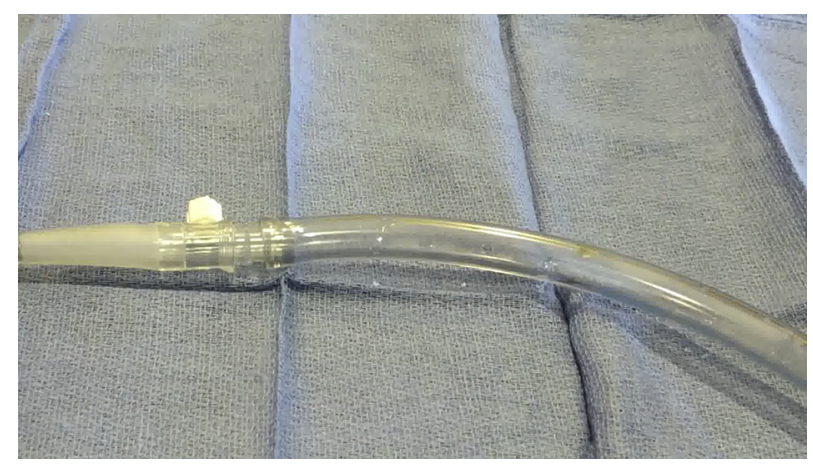

VIDEO 1. Video available at: https://www.jtcvs.org/article/S0022 5223(19)30475-1/fulltext.

Video 1). The prototype used here can accommodate catheters up to $8 \mathrm{~F}$ in size. To avoid stagnant blood flow and thrombus formation during prolonged support, this cap may be exchanged for an obturator that occludes the lumen of the side arm and allows laminar flow through the body of the system (Figure 1, $C$ and $D$ ).

\section{Computational Modeling}

The geometries of cannulae and the ECMO adaptor were obtained from computer-aided design files. The blood flow domains in these devices were extracted from the computer-aided design files for analysis using ANSYS Design modeler 19.0 (ANSYS Inc, Canonsburg, Pa). Both the inlet and outlet were extended to obtain the fully developed flow and avoid boundary effects. Both structured and unstructured mesh elements were used for the fluid domains of each cannula design. Meshes in the regions with complex flow patterns or high shear stress were particularly refined; inflation layers were used in the regions adjacent to the cannula wall for more accurate prediction of boundary layer behavior. Mesh independence analysis was performed to ensure the mesh quality and satisfactory convergence. There were approximately 700,000 elements for each cannula geometry. The blood flow simulation was conducted using the commercial finite volume solver FLUENT 19.0 (ANSYS Inc). A blood flow rate of 4 LPM and an outlet pressure of $80 \mathrm{~mm} \mathrm{Hg}$ were used for the simulation. All walls were set to be no-slip and no-penetration. Blood was treated as a Newtonian fluid with a density of $1050 \mathrm{~kg} / \mathrm{m}^{3}$ and viscosity of $3.5 \mathrm{cP}$. The SIMPLE pressure-velocity coupling method was used to solve all equations with second-order accuracy. The SST k- $\omega$ model with low Reynolds number corrections was used. The following velocity-pressure boundary conditions were prescribed: (1) uniform flows at the inlet and (2) uniform pressure at the outlet. Gravity was not considered in the simulation. Solutions were considered converged when (1) residuals were smaller than $10^{-5}$, (2) the inlet pressure approached steady values, and (3) the difference between the outlet and inlet flow rates was smaller than $5 \%$. Velocity vector fields, contours, and streamlines were extracted and plotted after simulations had converged. This process was performed for a conventional $19 \mathrm{~F}$ arterial cannula (Maquet, Rastatt, Germany), the ECMO adaptor, and the ECMO adaptor with access side arm obturator. The computational fluid dynamics analysis was then performed with a 19F EndoReturn arterial cannula (Edwards Lifesciences, Irvine, Calif) to test against a commercially available cannula that allows for access through a side port.

\section{In Vitro Testing}

To test the effect of accessing the ECMO adaptor with wires or catheters, a mock loop was created with a $25 \mathrm{~F}$ venous cannula (Maquet, Rastatt, Germany), a Cardiohelp integrated oxygenator and pump (Maquet), and varying sizes $(15 \mathrm{~F}-23 \mathrm{~F})$ of arterial cannulae (Maquet). The venous and arterial cannulae were placed in a glycerol solution bath with a viscosity of $3.4 \mathrm{cP}$ to model blood. With the ECMO adaptor unaccessed, flow, 


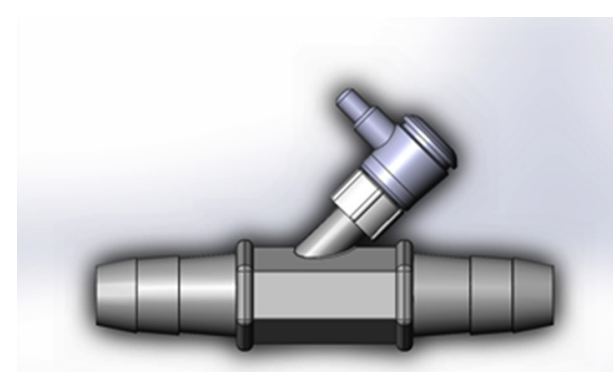

A

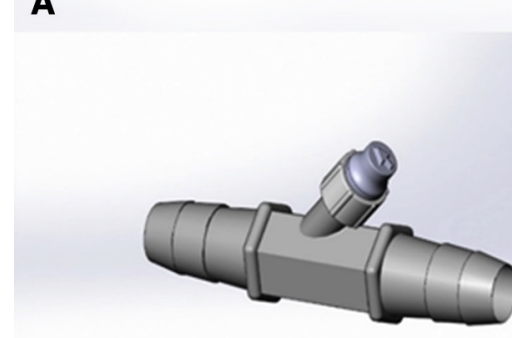

C

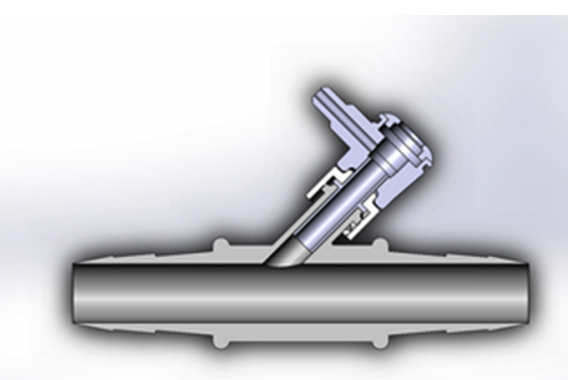

B

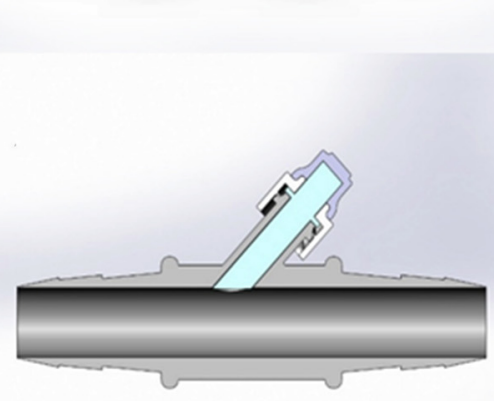

D

FIGURE 1. A and B, The novel adaptor system with a cylindrical tube and tapered ends that may be reversibly inserted into ECLS tubing. An angled side port interfaces with a hemostatic cap that allows passage of wires and catheters, as well as de-airing. $\mathrm{C}$ and $\mathrm{D}$, The cap exchanged for an obturator to avoid stagnant blood flow and thrombus formation during prolonged support.

arterial pressure, and venous pressure were measured at 2500, 3000, 3500, and 4000 RPM. Arterial pressure was defined as the pressure measured postpump, and venous pressure was defined as the pressure measured prepump. Values for flow, arterial pressure, and venous pressure were obtained from the Cardiohelp console and recorded for each set of conditions. Per the manufacturer of the Cardiohelp system, flow is measured by ultrasound. When flow is greater than $1 \mathrm{~L} / \mathrm{min}$, the accuracy of the measurement is $\pm 7 \%$ of the measured value. Likewise, for pressures between -500 and $-151 \mathrm{~mm} \mathrm{Hg}$, the accuracy of the measurement is $\pm 7 \%$ of the displayed value. For values between -150 and $+249 \mathrm{~mm} \mathrm{Hg}$, the measuring accuracy is $\pm 10 \mathrm{~mm} \mathrm{Hg}$. For pressures between +250 and $900 \mathrm{~mm} \mathrm{Hg}$, the accuracy of the measurement is $\pm 7 \%$ of the value. This was repeated for $15 \mathrm{~F}, 17 \mathrm{~F}$, $19 \mathrm{~F}, 21 \mathrm{~F}$, and $23 \mathrm{~F}$ arterial cannulae. A 0.035 " wire was then placed through the ECMO adaptor until the wire extended beyond the tip of the arterial cannula, and these measurements were repeated. A $5 \mathrm{~F}$ catheter was then placed through the adaptor, and the measurements were repeated. The wire and catheter were then removed, and the experiments were repeated 3 times. Mean values for each parameter measured and standard deviation from the mean were calculated on the basis of measured values.

\section{In Vivo Testing}

In vivo swine studies were performed with approval by the Institutional Animal Care and Use Committee, and all animals received humane care in compliance with the Guide for the Care and Use of Laboratory Animals. Yorkshire swine (40-60 kg, $\mathrm{n}=2$ ) were used for this study. Under ultrasound and fluoroscopic guidance, a $25 \mathrm{~F}$ venous drainage cannula (Maquet) was percutaneously placed in the femoral vein with the tip of the cannula at the caudal cavo-atrial junction. An arterial cannula was then percutaneously placed in the carotid artery $(17 \mathrm{~F}, \mathrm{n}=1)$ or the femoral artery (19F, $\mathrm{n}=1$ ). The tip of carotid arterial cannula was $1 \mathrm{~cm}$ proximal to origin of the carotid artery. The tip of the femoral arterial cannula was $2 \mathrm{~cm}$ proximal to the origin of the common iliac artery. The arterial and venous cannulae were connected to an ECMO circuit with a Quadrox oxygenator and Rotaflow centrifugal pump (Maquet). After achieving full ECMO flow (2.5-3.0
LPM), the adaptor was spliced into the circuit adjacent to the arterial cannula.

A 0.035 " Benston wire was placed through the adaptor and guided into the left ventricle. A $5 \mathrm{~F}$ pigtail catheter was then placed into the left ventricle, and a ventriculogram was performed. The catheter was repositioned to the aortic root, and descending aorta and angiographic images were obtained to demonstrate feasibility. The catheter was then exchanged for a $7 \mathrm{~F}$ coronary guide catheter. The left main coronary artery was then engaged, and the ability to perform coronary angiography was tested. A 0.014 " wire was then placed into the left anterior descending and circumflex coronary arteries to demonstrate the ability to access the coronary circulation.

\section{Outcomes and Statistical Analysis}

The primary outcome of this study was to evaluate the feasibility of the novel ECMO adaptor to allow for arterial access through an ECMO circuit for diagnostic and interventional endovascular procedures. This was evaluated with in vitro testing of flow and pressure after inserting a 0.035 " wire and $5 \mathrm{~F}$ catheter through the adaptor. The relationship between pump speed and flow rate was calculated for each cannula size (15F-23F) with the wire or catheter in place using least-squares linear regression. Feasibility was further evaluated by performing various diagnostic procedures in vivo.

Continuous variables are presented as means and standard deviations, and were compared with the Student $t$ test.

\section{RESULTS}

\section{Computational Modeling}

Blood flow through a $19 \mathrm{~F}$ arterial cannula was laminar with no discrete areas of flow stagnation (Figure 2, $A-C$ ). When assessing flow using a commercially available cannula with a side port (EndoReturn arterial cannula), flow remained laminar through the arterial cannula, but 


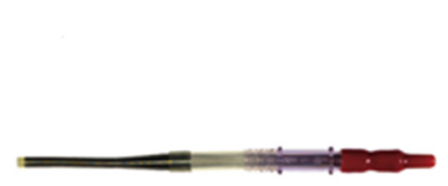

A

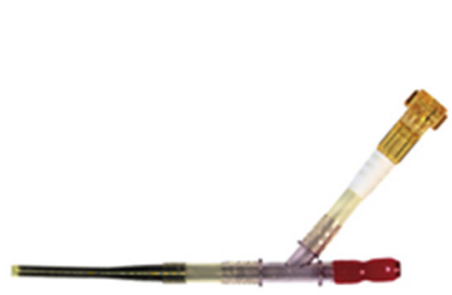

D
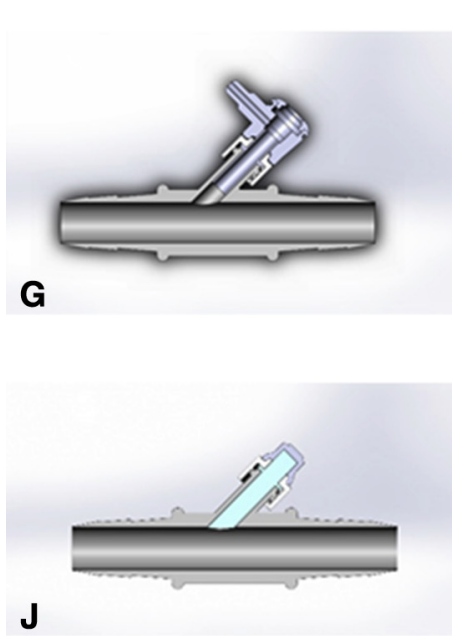
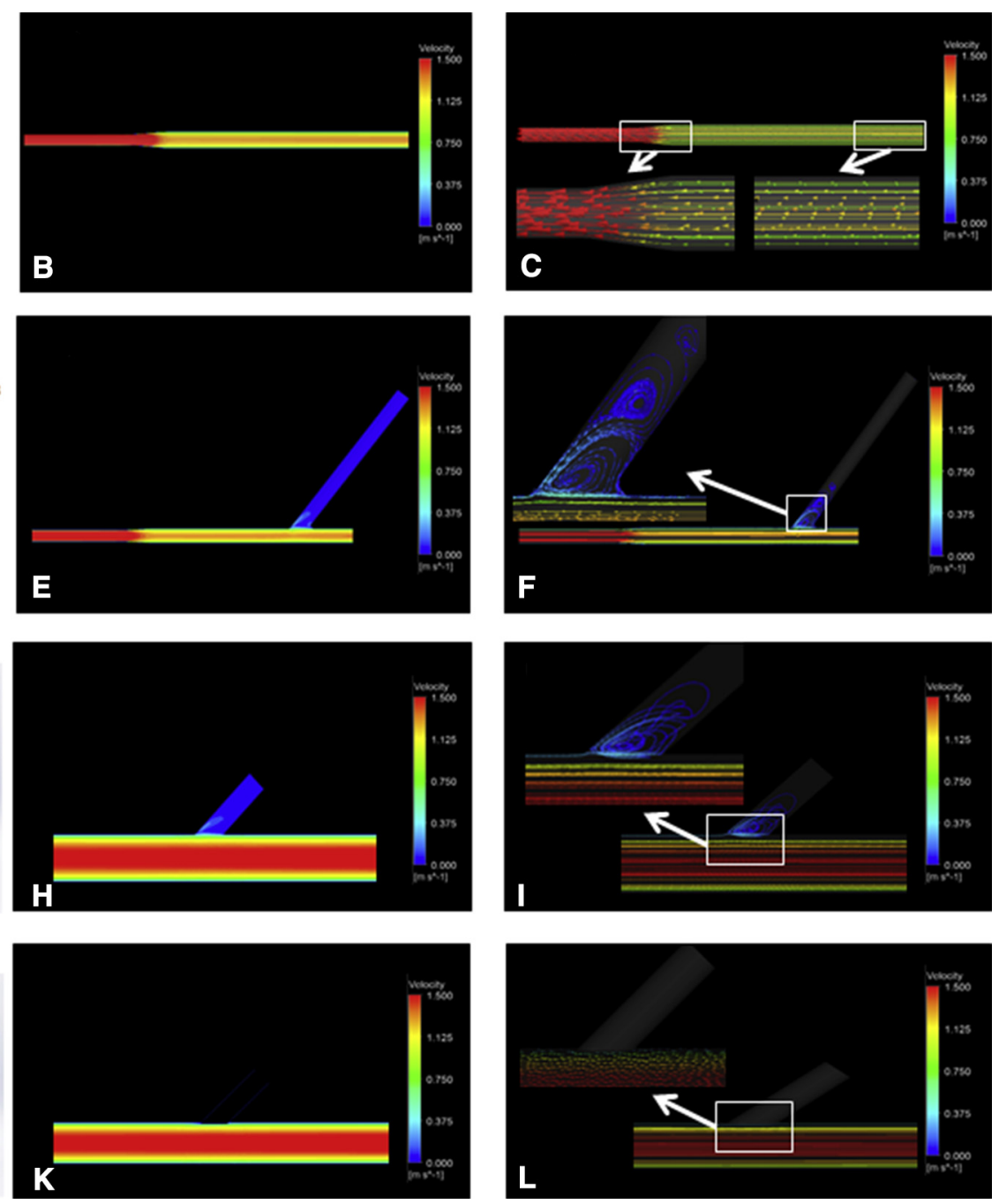

FIGURE 2. A-C, Computational fluid dynamics (CFD) analysis of blood flow through a 19F Maquet (Rastatt, Germany) arterial cannula demonstrating laminar flow with no discrete areas of flow stagnation. D-F, CFD analysis of blood flow through a commercially available cannula with a side port (EndoReturn arterial cannula; Edwards Lifesciences, Irvine, Calif), demonstrating considerable flow stagnation within the long side access port. G-I, CFD analysis of flow through the ECMO adaptor demonstrating laminar flow but stagnation in the access port. J-L, CFD analysis of flow through the ECMO adaptor with insertion of an obturator in the side arm port demonstrating laminar flow without stagnation.

considerable flow stagnation was observed within the long side access port (Figure 2, D-F). Within the side access port, particle flow decreased from $0.4 \mathrm{~m} / \mathrm{s}$ at the take-off from the cannula to less than $0.01 \mathrm{~m} / \mathrm{s}$ in the distal $81 \%$ of the side port. Flow through the novel ECMO adaptor demonstrated similar findings (Figure 2, G-I) with flow stagnation in the access port and particle flow less than $0.01 \mathrm{~m} / \mathrm{s}$ in the distal $33 \%$ of the access port. However, upon insertion of an obturator in the side arm port, laminar flow without stagnation was observed (Figure 2, J-L).

\section{In Vitro Testing}

By using a small arterial cannula (15F) at $3500 \mathrm{RPM}$ in mock loop experiments, a flow of $3.21 \pm 0.02 \mathrm{LPM}$ was achieved. With a $15 \mathrm{~F}$ cannula, insertion of a 0.035 " wire through the ECMO adaptor resulted in a $15 \%$ decrease in flow from $3.21 \pm 0.02$ to $2.73 \pm 0.00 \operatorname{LPM}(P<.001)$. There was an associated $9 \%$ increase in arterial pressure from $255 \pm 0.00 \mathrm{~mm} \mathrm{Hg}$ to $277 \pm 0.00 \mathrm{~mm} \mathrm{Hg}$. Insertion of a $5 \mathrm{~F}$ catheter resulted in a $24 \%$ decrement in circuit flow from $3.21 \pm 0.02 \mathrm{LPM}$ to $2.34 \pm 0.00 \mathrm{LPM}(P<.001$, Figure 3$)$. There was an associated $16 \%$ increase in arterial pressure from $255 \pm 0 \mathrm{~mm} \mathrm{Hg}$ to $295 \pm 1 \mathrm{~mm} \mathrm{Hg}$.

When using a $23 \mathrm{~F}$ arterial cannula at $3500 \mathrm{RPM}$, a flow of $4.72 \pm 0.05 \mathrm{LPM}$ was achieved. After inserting the wire, there was only an $3 \%$ decrement in circuit flow from $4.72 \pm 0.05 \mathrm{LPM}$ to $4.58 \pm 0.00 \mathrm{LPM}$ with a $5 \%$ increase in arterial pressure $(P<.001$ for all values $)$. Moreover, after inserting the catheter, there was only a $5 \%$ decrement in circuit flow to $4.48 \pm 0.00 \mathrm{LPM}$ with a $10 \%$ increase 

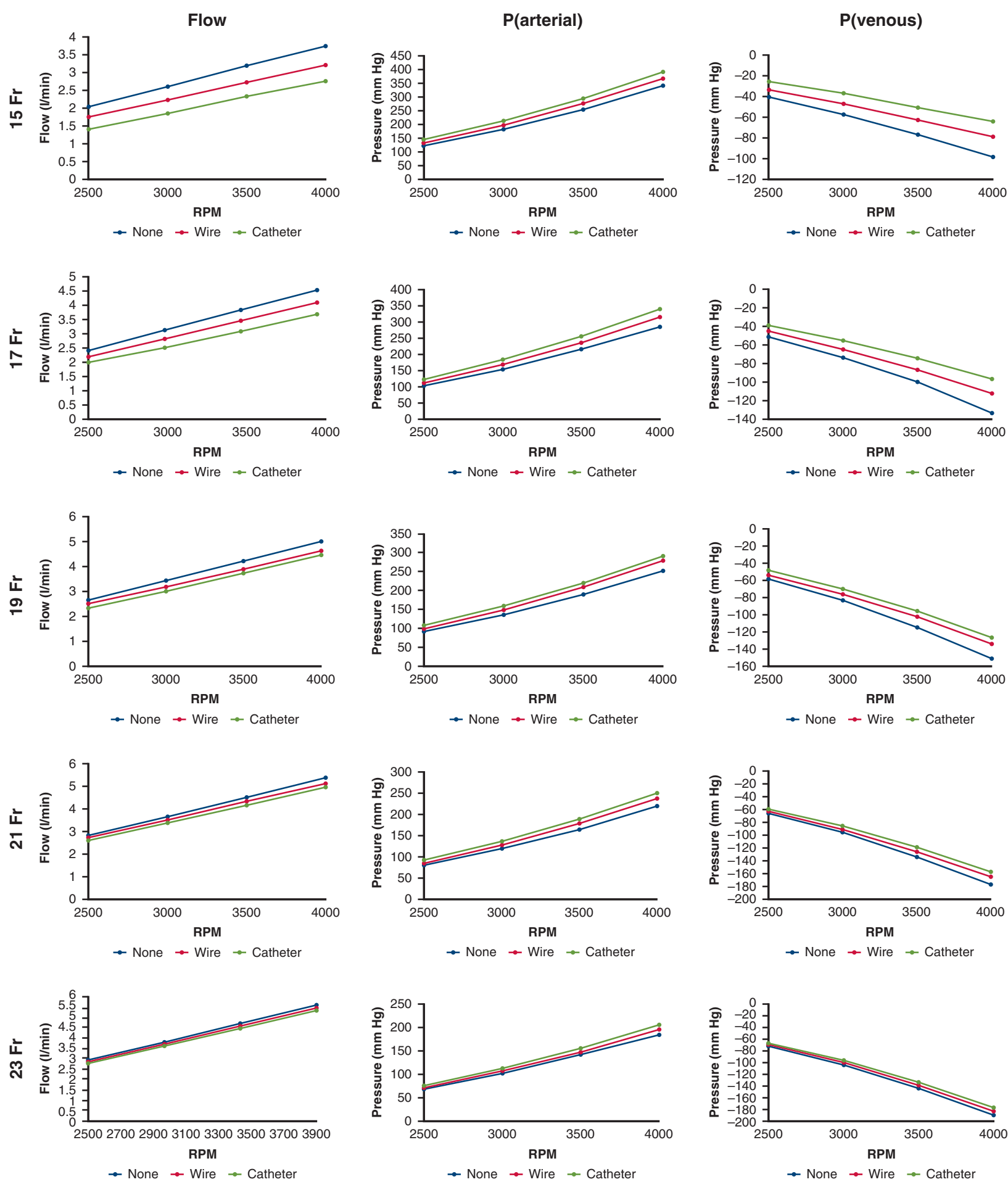

FIGURE 3. A mock loop was used to measure flow and pressure at various pump speeds in an ECLS circuit to assess changes with insertion of guidewires and catheters through the adaptor. Flow, arterial pressure ( $\mathrm{P}($ arterial $))$ and venous pressure $(\mathrm{P}($ venous $))$ with in vitro mock loop set-up are presented. Values represent the mean of 3 measurements, and error bars represent the standard deviation of the mean. $P<.05$ at all points.

in arterial pressure $(P<.001$ for all values). By using the flow and speed data points collected, a least-squares linear regression equation was created with a $21 \mathrm{~F}$ arterial cannula and either the catheter or inserted wire $\left(\right.$ Flow $_{i}=-1.3+0.0016 \times$ Speed $\left._{i}, r^{2}=0.99, P<.001\right)$. A modest increase in pump speed from 3500 to 3631 RPM would normalize flow to the nonaccessed state. Similar trends were seen with 17F, 19F, and 23F cannulae, 


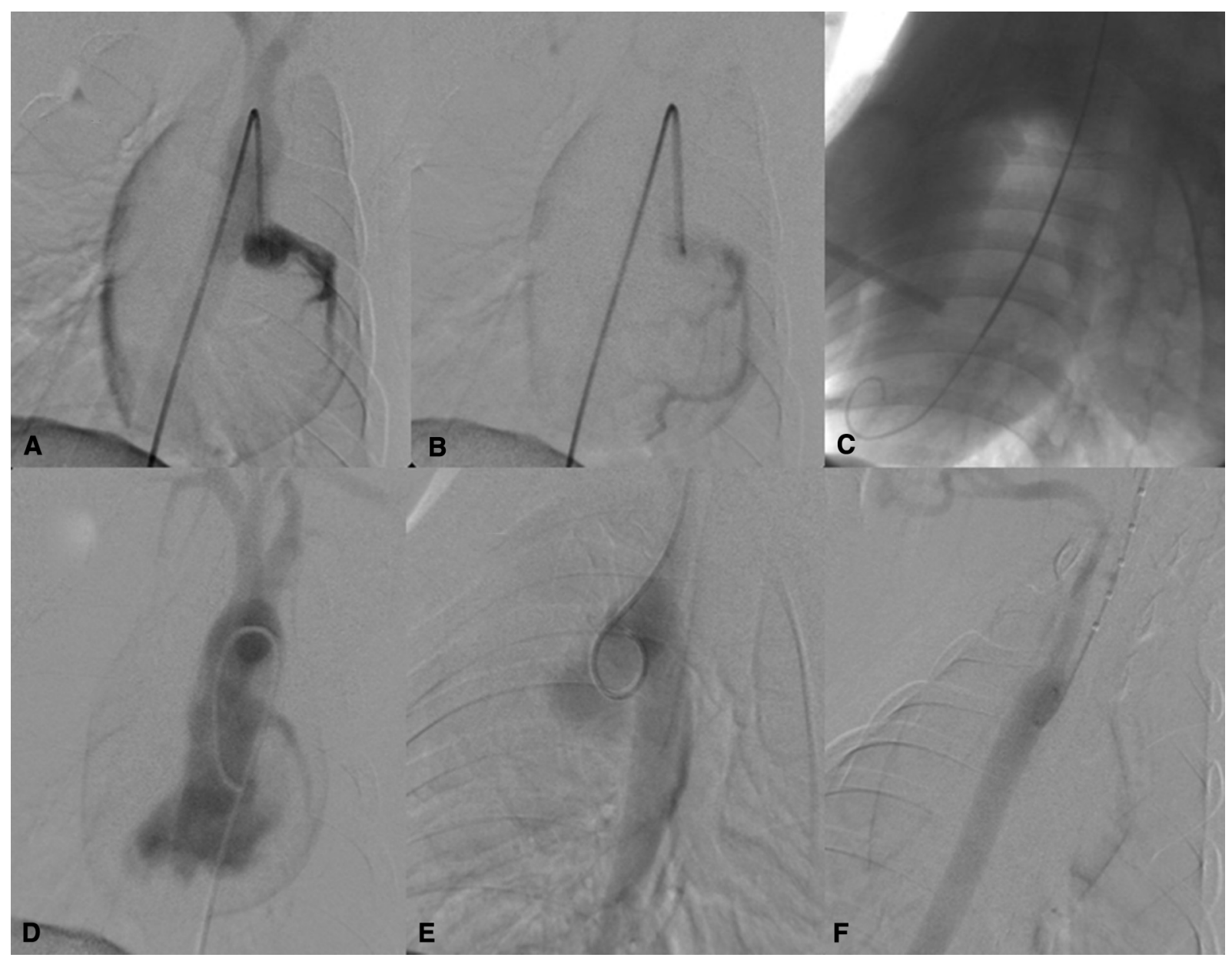

FIGURE 4. In vivo swine model demonstrating the feasibility of performing various procedures through an ECLS circuit using a novel adaptor system including (A and B) coronary angiography, (C and D) left ventriculography, (E) aortic root and ascending aortography, and (F) descending aortography.

with intermediate flow and pressure changes with the wire or catheter inserted.

\section{In Vivo Testing}

After successfully placing the swine on venoarterial ECMO and splicing the ECMO adaptor adjacent to the arterial line, a 0.035 " wire, and $7 \mathrm{~F}$ guide catheter were successfully advanced into the arterial system. Coronary angiography (Figure 4, $A$ and $B$ ), left ventriculography (Figure 4, $C$ and $D$ ), aortic root angiography (Figure $4, E$ ), and descending thoracic aortography (Figure $4, F$ ) were successfully performed. In these short-term experiments, no thrombus formation was noted inside or around the adaptor system.

\section{DISCUSSION}

To date, ECLS circuits have largely been used to support patients with life-threatening cardiac or pulmonary failure. In this study, a novel modular adaptor system that can be easily inserted and removed from an ECLS circuit is presented. These data demonstrate that a novel adaptor system can enable the performance of diagnostic and therapeutic procedures in patients requiring ECLS by using the circuit itself as an access point to the cardiovascular system.

This adaptor system could have broad application in clinical practice. For example, ECMO is being more frequently used as an adjunct to cardiopulmonary resuscitation (extracorporeal cardiopulmonary resuscitation) in patients experiencing cardiac arrest and has resulted in improved survival in patients with cardiac arrest. ${ }^{7,8}$ Previous data suggest that clinically significant coronary disease is common in survivors of out-of-hospital cardiac arrest and that immediate coronary angiography and intervention are beneficial. ${ }^{9-13}$ More recent data suggest that early cardiac catheterization is also beneficial in patients undergoing ECPR. ${ }^{7,14-16}$ This adaptor system would facilitate rapid cardiac catheterization in this patient population, and no additional arterial access would be required.

Furthermore, temporary mechanical support is increasingly being used in patients undergoing high-risk PCI, a practice that is likely to expand in scope. ${ }^{17}$ Commonly 
used systems for temporary support during high-risk PCI, such as the Impella 2.5 device (Abiomed, Danvers, Mass), can provide only partial hemodynamic support and do not provide gas exchange. In addition, 1 arterial access site is required for the temporary support device and another is required for the cardiac catheterization. Because it can provide full hemodynamic support as well as gas exchange, ECMO may be desirable as a support platform for highrisk PCI. Our adaptor system would allow 1 arterial access site to suffice for both mechanical support and intervention.

Other potential applications also exist. For patients on venoarterial ECMO with left ventricular distention, this adaptor may allow for insertion of an intra-aortic balloon pump or even a specially designed left ventricular vent. For patients on venovenous ECMO, this adaptor may serve as an access site for right heart catheterization or instillation of therapeutics, such as thrombolytics, into the pulmonary circulation. When used in venovenous ECMO, this device would be used exclusively on the arterial/inflow limb of the circuit. Otherwise, there would be a risk of entraining air into the circuit, which could have catastrophic consequences. Last, because this adaptor system can provide endovascular wire access through and existing ECLS circuit, it may facilitate percutaneous closure of arterial cannulation sites using large-bore arterial closure devices.

This novel modular adaptor system has a number of features that facilitate its use in these scenarios. Specifically, it allows hemostatic insertion and removal of catheters and wires, which are directed toward the patient through its design. Our data suggest that at clinically relevant pump speeds, insertion of wires and catheters through the adaptor system results in only a clinically insignificant decrease in flow and increase in arterial pressure. Further, the small decrements in flow that are observed can be countered by increasing pump speeds within acceptable limits.

In addition, many patients undergo cannulation at centers that do not routinely care for patients who require ECMO with subsequent transfer to centers that specialize in the care of ECLS patients. Centers that do not routinely initiate ECLS may lack specialized cannulas or equipment. Because our adaptor can be easily inserted into an ECLS circuit with only brief interruption of flow, it can be integrated into the circuit after transfer of a patient to a specialized center so that the patient could then undergo diagnostic and therapeutic interventions as indicated. In addition, if more than 1 access site is required for a complex procedure, additional adaptors potentially could be inserted in serial in the circuit.

Of note, areas of stagnant blood flow in an ECLS circuit may result in thrombus formation with potentially catastrophic complications, including stroke or visceral ischemia. For these reasons, we have incorporated an obturator that can be reversibly exchanged with an access port in our design. With the obturator in place, stagnant flow is eliminated and laminar flow through the adaptor is observed. However, thrombus formation may occur around any type of connector in an ECMO circuit. As an alternative, the adaptor may be removed from the circuit entirely after completion of the procedure. Removing the adaptor would mitigate this problem, but requires a brief interruption in circuit flow and removal of a small portion of tubing.

\section{CONCLUSIONS}

This novel adaptor system successfully enables endovascular access through an ECLS circuit. Although longerterm testing using clinical grade materials is required, this technology may transform ECLS from a purely supportive strategy to a platform for endovascular intervention and may have broad application in a number of common settings where ECLS is used, including high-risk PCI, cardiac arrest, and pulmonary embolus.

\section{Conflict of Interest Statement}

D.J.K. discloses intellectual property and ownership interest in ECMOTEK LLC. C.P., G.J.B., B.P.G., and Z.N.K. disclose intellectual property for an ECMO cannula. Z.N.K. receives consulting fees from Breethe, Inc. B.P.G. and Z.W. disclose ownership interested in Breethe, Inc. All other authors have nothing to disclose with regard to commercial support.

\section{References}

1. Hill JD, O'Brien TG, Murray JJ, Dontigny L, Bramson ML, Osborn JJ, et al. Prolonged extracorporeal oxygenation for acute post-traumatic respiratory failure (shock-lung syndrome). Use of the Bramson membrane lung. $N$ Engl J Med. 1972;286:629-34.

2. Squiers JJ, Lima B, DiMaio JM. Contemporary extracorporeal membrane oxygenation therapy in adults: fundamental principles and systematic review of the evidence. J Thorac Cardiovasc Surg. 2016;152:20-32.

3. Australia and New Zealand Extracorporeal Membrane Oxygenation (ANZ ECMO) Influenza Investigators, Davies A, Jones D, Bailey M, Beca J, Bellomo R, et al. Extracorporeal membrane oxygenation for 2009 influenza A(H1N1) acute respiratory distress syndrome. JAMA. 2009;302:1888-95.

4. Sauer CM, Yuh DD, Bonde P. Extracorporeal membrane oxygenation use has increased by $433 \%$ in adults in the United States from 2006 to 2011. ASAIO J. 2015;61:31-6.

5. Maxwell BG, Powers AJ, Sheikh AY, Lee PH, Lobato RL, Wong JK. Resource use trends in extracorporeal membrane oxygenation in adults: an analysis of the nationwide inpatient sample 1998-2009. J Thorac Cardiovasc Surg. 2014;148:416-21.

6. McCarthy FH, McDermott KM, Kini V, Gutsche JT, Wald JW, Xie D, et al. Trends in U.S. extracorporeal membrane oxygenation use and outcomes: 20022012. Semin Thorac Cardiovasc Surg. 2015;27:81-8.

7. Kehrl T, Kaczorowski DJ. Extracorporeal life support for cardiopulmonary resuscitation for adults: evolving evidence. ASAIO J. 2016;62:364-9.

8. Peigh G, Cavarocchi N, Hirose H. Saving life and brain with extracorporeal car diopulmonary resuscitation: a single-center analysis of in-hospital cardiac arrests. J Thorac Cardiovasc Surg. 2015;150:1344-9.

9. Spaulding CM, Joly LM, Rosenberg A, Monchi M, Weber SN, Dhainaut JF, et al Immediate coronary angiography in survivors of out-of-hospital cardiac arrest. N Engl J Med. 1997;336:1629-33.

10. Dumas F, Cariou A, Manzo-Silberman S, Grimaldi D, Vivien B, Rosencher J, et al Immediate percutaneous coronary intervention is associated with better survival after out-of-hospital cardiac arrest: insights from the PROCAT (Parisian Region Out of hospital Cardiac ArresT) registry. Circ Cardiovasc Interv. 2010;3:200-7.

11. Strote JA, Maynard C, Olsufka M, Nichol G, Copass MK, Cobb LA, et al. Comparison of role of early (less than six hours) to later (more than six hours) or no 
cardiac catheterization after resuscitation from out-of-hospital cardiac arrest. Am J Cardiol. 2012;109:451-4.

12. Zanuttini D, Armellini I, Nucifora G, Carchietti E, Trillò G, Spedicato L, et al. Impact of emergency coronary angiography on in-hospital outcome of unconscious survivors after out-of-hospital cardiac arrest. Am J Cardiol. 2012;110:1723-8.

13. Camuglia AC, Randhawa VK, Lavi S, Walters DL. Cardiac catheterization is associated with superior outcomes for survivors of out of hospital cardiac arrest: review and meta-analysis. Resuscitation. 2014;85:1533-40.

14. Stub D, Bernard S, Pellegrino V, Smith K, Walker T, Sheldrake J, et al. Refractory cardiac arrest treated with mechanical CPR, hypothermia, ECMO and early reperfusion (the CHEER trial). Resuscitation. 2015;86:88-94.

15. Yannopoulos D, Bartos JA, Martin C, Raveendran G, Missov E, Conterato M, et al. Minnesota resuscitation consortium's advanced perfusion and reperfusion cardiac life support strategy for out-of-hospital refractory ventricular fibrillation. J Am Heart Assoc. 2016;5.

16. Yannopoulos D, Bartos JA, Raveendran G, Conterato M, Frascone RJ, Trembley A, et al. Coronary artery disease in patients with out-of-hospital refractory ventricular fibrillation cardiac arrest. J Am Coll Cardiol. 2017; 70:1109-17.

17. Khera R, Cram P, Vaughan-Sarrazin M, Horwitz PA, Girotra S. Use of mechanical circulatory support in percutaneous coronary intervention in the United States. Am J Cardiol. 2016;117:10-6.

Key Words: extracorporeal life support, extracorporeal membrane oxygenation, endovascular, mechanical support 